\title{
ZU EINIGEN STRUKTURELLEN EIGENSCHAFTEN VON NOMINALISIERUNGEN IM DEUTSCHEN
}

\section{NOMINALISIERUNGSTENDENZEN IM DEUTSCHEN}

Wie in den meisten Literatursprachen zeigt sich auch in der deutschen Sprache eine allgemeine Tendenz zur Nominalisierung. ' Neben dem Ausbau von hochgradig komprimierten Nominalgruppen, deren Gliedkern oft ein Verbalstraktum ist, gelten die Funktionsverbgefüge als "die zweite Erscheinungsweise der Entwicklungstendenz der deutschen Sprache zum Nominalisierungsstil"2 oder sogar "als Hauptträger nominalisierender Tendenzen" ${ }^{3}$ in der deutschen Sprache.

Im Aufsatz sollen die folgenden Annahmen vorgeführt werden: Nominalisierungen mit Verbalabstraktum als Gliedkern können sich in der deutschen Gegenwartssprache in zwei verschiedene Richtungen entwickeln.

In freien Fügungen verlieren die Verbalabstrakta an verbalen Eigenschaften und können mit zunehmender Lexikalisierung in stärkerem Maße ins nominale Paradigma eingebaut werden. Im Vergleich $\mathrm{zu}$ den entsprechenden Nebensätzen sind die Nominalisierungen als geschwächte Konstruktionen ${ }^{4}$ anzusehen.

In Funktionsverbgefügen verlieren die Verbalabstrakta an nominalen Eigenschaften und werden mit zunehmender Lexikalisierung der Fügung in stärkerem Maße

1 Vgl. Daniels 1963, v. Polenz 1963, Engelen 1968, Heringer 1968, Bausch 1964: 223, zitiert nach Heringer 1968: 120. u.a.

2 Vgl. v. Polenz 1985: 113.

3 Vgl. Daniels 1963: 10.

4 Die Begriffe 'verstärkte Konstruktion' und 'geschwächte Konstruktion' sind natürlichkeitstheoretischer Natur und werden in Orešnik 1990a: 85-87 und Orešnik et alii 1990b: 5-7 erklärt. Die Grundannahme besagt folgendes: Am Anfang (d.h. im Stadium, in welchem eine Konstruktion nur als eine syntaktische Variante eines anderen Ausdrucks zu werten und noch nicht grammatikalisiert ist) behaupten sich verstärkte Konstruktionen vorzugsweise unter relativ komplizierten (markierten) grammatischen Verhältnissen und verbreiten sich in ihrer späteren Entwicklung unter Umständen auch unter weniger komplizierten grammatischen Verhältnissen. Geschwächte Konstruktionen dagegen behaupten sich am Anfang vorzugsweise unter relativ einfachen (weniger markierten) grammatischen Verhältnissen und verbreiten sich später möglicherweise auch unter weniger einfachen grammatischen Verhältnissen. 
ins verbale Paradigma eingebaut. Im Vergleich zu den entsprechenden einfachen Verben sind solche Gefüge als verstärkte Konstruktionen ${ }^{5}$ anzusehen.

In Nominalisierungsverbgefügen sind beide Entwicklungstendenzen ausgeprägt, d.h. sowohl Tendenzen zum Einbau ins nominale Paradigma als auch Tendenzen zum Einbau ins verbale Paradigma. Im Vergleich zu den entsprechenden einfachen Verben zeigen solche Gefüge Eigenschaften verstärkter Konstruktionen. ${ }^{6}$

\section{ZU DEN BEGRIFFEN 'FREIE FÜGUNG', 'FUNKTIONS- VERBGEFÜGE' UND 'NOMINALISIERUNGSVERBGEFÜGE'}

Unter freien Fügungen verstehen wir jene verbal-nominale Verbindungen, in denen sowohl das Verb als auch das Verbalabstraktum semantisch und syntaktisch selbständig ist. Die Ungebundenheit und Variabilität der beiden Kombinationsglieder zeigt sich in ihren unterschiedlichen semantischen und syntaktischen Wertigkeiten, die von ihrer Kookurrenz nicht beeinträchtigt werden und sich zu einer Prädikation ergänzen. ${ }^{7}$ Daher ist

a) eine Umformung einer Nominalisierung in einen Nebensatz oder Infinitvsatz bei Bebehaltung des Verbs der freien Fügung möglich,

b) das Verb oder das Substantiv der freien Fügung durch Synonyme, Antonyme oder beliebige andere Lexeme ersetzbar und

c) das Substantiv der freien Fügung anaphorisierbar, ohne daß sich die Bedeutung des Verbs der freien Fügung verändert. ${ }^{8}$

(1) Sie müssen in Ihrer Argumentation, wenn Sie die ersatzlose STREICHUNG des Paragraphen 218 durchsetzen wollen, klartun, daß der Gesetzgeber in diesem Falle nicht die Pflicht hat, das werdende Leben als Bestandteil unseres Lebens insgesamt zu schützen."

(2) Sie müssen in Ihrer Argumentation, wenn Sie es durchsetzen wollen, daß der Paragraph 218 ersatzlos gestrichen wird, klartun, daß der Gesetzgeber in diesem Falle nicht die Pflicht hat, das werdende Leben als Bestandteil unseres Lebens insgesamt zu schützen.

5 Vgl. die vorherige Anmerkung.

6 Ausführlicher über diese Annahmen und die dazu gehörenden statistischen Ergebnisse in Petrič 1990 bzw. Petrič 1992 und in Petrič 1991 bzw. 1993.

7 Vgl. Köhler 1985: 20f.

8 Vgl. Köhler 1985: $21 \mathrm{f}$.

9 Aus dem Textbuch Heutiges Deutsch 1974, Text 'Moral 71. Zum Beispiel Abtreibung, S. 369'. 
(3) Sie müssen in Ihrer Argumentation, wenn Sie die STREICHUNG des Paragraphen 218 / Verabschiedung des Paragraphen 218 / ihren Plan / ... durchsetzen wollen, klartun, daß ...

(4) Sie müssen in Ihrer Argumentation, wenn Sie die ersatzlose STREICHUNG des Paragraphen 218 durchsetzen / erreichen / ... wollen, klartun, daß ...

(5) Sie müssen in Ihrer Argumentation, wenn Sie es / das / etwas / ... durchsetzen wollen, klartun, daß ...

Grundlegende Eigenschaften der Bestandteile einer freien Fügung sind aus den Beispielen (1)-(5) ersichtlich.

Die Meinungen darüber, was ein Funktionsverb bzw. was ein Funktionsverbgefüge ist und damit, welche Strukturtypen dazu gehören, sind bekanntlich geteilt. Die folgenden Strukturtypen ${ }^{10}$ werden oft als Funktionsverbgefüge aufgeführt:

\subsection{Typ V + PP}

(6) zur Anwendung kommen, zum Abschluß bringen, in Kenntnis setzen.

\subsection{Typ V + Akk}

Subtyp 2a: (7) Anwendung finden, Einbuße erfahren (erleiden);

Subtyp 2b: (8) Kritik üben, Beitrag leisten, Antwort geben.

2.3 Typ $\mathbf{V}+\mathbf{N o m}$

(9) Zwischen den Delegierten besteht keine Übereinstimmung.

(10) Der Umtausch erfolgt gegen Vorlage der Quittung.

2.4 Typ $\mathbf{V}+$ Dat

(11) jemanden einer Prüfung unterziehen, etwas unterliegt der Kontrolle.

\subsection{Typ V + Gen}

Subtyp 5a: (12) der Klärung bedürfen;

Subtyp 5b: (13) der Meinung (Ansicht...) sein/bleiben.

Wir wollen die Typen 2.1 (6) und 2.2a (7) als Funktionsverbgefüge (FVG) bezeichnen, ${ }^{11}$ die Typen $2.2 \mathrm{~b}$ bis $2.5 \mathrm{~b}(8)-(13)$ in Anlehnung an v. Polenz ${ }^{12}$ hingegen als Nominalisierungsgefüge (NVG). Das Verb im FVG soll als Funktionsverb (FV) und das Substantiv im FVG als Funktionsnomen (FN) bezeichnet werden. Das Verb im NVG wird als Nominalisierungsverb (NV) und das Substantiv im NVG als Nomininalisierungsnomen (NN) bezeichnet.

FVG unterscheiden sich von NVG im wesentlichen dadurch,

a) daß sie auch noch "prädikative Zusatzfunktionen" erfüllen, die im Bereich der Kausativität, der Aktionsarten und der Passivität zu suchen sind, ${ }^{13}$

10 Terminologisch haben wir sie leicht verändert von Helbig 1979: 275 und So 1991: 9-10 übernommen.

11 So 1991: 11-13.

12 v. Polenz 1987: 170. 
b) durch die stärkere semantische Gebundenheit der Kombinationspartner aneinander und die größere syntaktische Festigkeit der Konstruktion, die sich anhand verschiedener formaler Merkmale offenbart.

Ein- und dasselbe Verbalabstraktum kann einmal in einer freien Fügung (14), ein anderes Mal in einem Nominalisierungsverbgefüge (16) oder auch in einem Funktionsverbgefüge (18) auftreten.

(14) In der Ferne bemerkte er eine BEWEGUNG.

(15) In der Ferne bemerkte er, daß sich etwas bewegte.

(16) Ja, euer Meerschweinchen hat ja wenigstens mehr BEWEGUNG.

(17) Ja, euer Meerschweinchen bewegt sich ja wenigstens mehr.

(18) Der Zug setzte sich wider Erwarten in BEWEGUNG.

(19) Der Zug bewegte sich wider Erwarten.

(20) Der Zug begann sich wider Erwarten zu bewegen.

\section{VERBALABSTRAKTA ALS VERSTÄRKTE WORTBIL- DUNGSKONSTRUKTIONEN}

Der Gliedkern einer Nominalisierung ist im Vergleich zu seiner verbalen Basis eine verstärkte Form. ${ }^{14}$ Diese Behauptung kann folgendermaßen begründet werden:

a) Dem Verbalstamm wird beim Ableitungsvorgang meist ein (oder sogar mehr als ein) Morphem hinzugefügt. Der Kern einer Nominalisierung ist demnach komplizierter gebaut als der entsprechende Verbstamm:

(21) Verwandl + ung, Ge + red + e.

b) Der Kern einer Nominalisierung behält meist nur bestimmte Bedeutungsvarianten der verbalen Basis bei und hat daher meistens weniger Bedeutungsvarianten als das zugrundeliegende Verb:

(22) erhalten : Erhaltung.

c) Verbalabstrakta sind überwiegend von "schweren" Verben abgeleitet, d.h. die zugrundeliegenden Verben sind kompliziertere Morphemkonstruktionen, daher längere Wörter, kommen relativ selten vor und sind vorwiegend Vollverben:

(23) sich einpassen ( $\longrightarrow$ die Einpassung).

13 v. Polenz 1987: 170ff.

14 In Petrič 1990 bzw. Petrič 1992 wurden aus der Annahme, Verbalabstrakta seien verstärkte Wortbildungskonstruktionen, Vorhersagen abgeleitet und mit statistischen Mitteln geprüft. Die Mehrzahl unserer Vorhersagen wurde bestätigt. 
d) Hinsichtlich ihrer Bedeutung gehören die Kerne der Nominalisierungen (die Verbalabstrakta) zu den kompliziertesten Substantiven. Dynamische Abstrakta sind komplizierter als statische Abstrakta und letztere komplizierter als Konkreta: ${ }^{15}$

(24) Hans < Feuer < Liebe < Überschreitung.

Nichtlexikalisierte Verbalabstrakta können nicht als prototypische Substantive eingestuft werden, denn

a) sie haben meist eine dynamische Bedeutung statt einer statischen, da ja Verben meist eine Handlung oder einen Vorgang zum Ausdruck bringen,

b) übernehmen im wesentlichen die verbale Valenz, ${ }^{16}$ wodurch der Anteil komplizierter Attributtypen (Objektgenitive, Präpostionalgruppen, Infinitivsätze, Nebensätze) größer ist als bei den prototypischen Substantiven,

c) können nicht pluralisiert werden. Bei Fehlen eines Objektgenitivs können perfektive Verbalabstrakta jedoch ein Pluralmorphem erhalten.

Im Laufe des Lexikalisierungsprozesses büßen Verbalabstrakta allerdings Eigenschaften ein, die sie von ihren zugrundeliegenden Verben ererbt haben, und erhalten dafür nominale:

a) Gewöhnlich entstehen neben der dynamischen (prozessualen) Lesart noch andere, meist statische Lesarten: die Bezeichung eines Zustandes, eines abstrakten Ergebnisses oder gar eines konkreten Ergebnisses des Prozesses, der durch den Verbalstamm des Verbalsubstantivs zum Ausdruck kommt.

b) In den sekundären statischen Lesarten sind die Verbalsubstantive in der Regel pluralisierbar.

c) Ein Zeichen der fortgeschrittenen Lexikalisierung ist auch die Veränderung von Akzentmustern, d.h. das Verbalabstraktum wird nach substanitvischen Akzentmustern betont und nicht nach verbalen Akzentmustern:

(25) die Übernahme - übernehmen.

d) Die Valenz, die ein Verbalsubstantiv von seinem Ausgangsverb ererbt, wird abgebaut und an nominale Valenzmuster angeglichen.

Im Gegenwartsdeutschen spielen die produktiven Ableitungsmuster mit dem nominalen Suffix -ung eine wichtige Rolle. In den FF unseres ersten Korpus ${ }^{17}$ haben sie

Vgl. Mayerthaler 1981: 17f.

16 Die Genitiv- oder Dativergänzung eines Verbs muß im Deutschen durch eine Periphrase an das Verbalabstraktum angeschlossen werden. Treten ein Subjekt und ein Akkusativobjekt gleichzeitig als Attribut zu einem Verbalabstraktum, muß das Subjekt ebenfalls periphrastisch wiedergegeben werden (von/durch-Phrase). Zur periphrastischen Strategie vgl. Toman 1987.

17 Das Korpus setzt sich zusammen aus den dre Diskussionen 'Meinung gegen Meinung. Fragen der Verkehrssicherheit', 'Schulklassengespräch mit Günter Grass', 'Moral 71. Zum Beispiel Abtreibung' in den Textbüchern Heutiges Deutsch 1971 und 1974 sowie aus dem Theaterstück 'Die Fische' von Peter Hacks (vgl. Petrič 1990 bzw. Petrič 1992). 
einen Anteil von mehr als $50 \%,{ }^{18}$ während die impliziten Ableitungen und die substantivierten Infinitive erst auf dem zweiten bzw. dritten Platz folgen. Auch in den FVG und NVG unseres zweiten Korpus ${ }^{19}$ haben die Ableitungen auf -ung den größten Anteil (etwa 32\% bzw. 39\%), obwohl dieser auffällig kleiner ist als in den FF unseres obigen Korpus. ${ }^{20}$ In FVG begann der Aufstieg der Ableitung auf -ung vor etwa 200 Jahren, ihre Verwendung wurde aber erst in der zweiten Hälfte des 19. Jahrhunderts und im 20. Jahrhundert richtig intensiv. ${ }^{21}$ Noch im 17. und 18. Jahrhundert bestand der nominale Teil der FVG viel häufiger aus substantivierten Infinitiven. Die Ableitungen auf -ung waren nämlich ihrer Häufigkeit nach erst an dritter Stelle - nach den Konversionen und impliziten Ableitungen. Mit dem Aufstieg der Ableitungen auf -ung gingen die relative Zunahme verbaler Basen und der prozentuale Rückgang adjektivischer Basen von Verbalabstrakta in FVG einher. ${ }^{22}$ Damit verbunden ist der größere Anteil an Ableitungen mit dynamischer Bedeutung in FVG. Da die Basisverben der Ableitungen auf -ung meist terminative Verben sind, ist auch der Anteil der terminativen Ableitungen größer. Warum wird in der deutschen Gegenwartssprache die Ableitung auf -ung gegenüber dem substantivierten Infinitiv bevorzugt, obwohl letzterer doch im Gegensatz zur ersteren von nahezu allen Verbtypen gebildet werden kann ? In der linguistischen Literatur werden verschiedene Gründe dafür angeführt. v. Polenz $^{23}$ weist darauf hin, daß die Ableitungen auf -ung im Gegensatz zu substantivierten Infinitiven genusneutrale Prädikatsbezeichnungen sind. So können substantivierte Infinitive im heutigen Deutschen im Gegensatz zu den Ableitungen auf -ung nicht artikellos verwendet werden. Schäublin ${ }^{24}$ zeigt in seiner Arbeit, daß der substantivierte Infinitiv in einer Reihe von Merkmalen dem Verb formal nähersteht als die Ableitung auf -ung. Den formalen Unterschieden entspricht auch eine inhaltliche Differenzierung. Die vielseitige kontextuelle Verwendbarkeit der Ableitungen auf -ung kann als eine Funktion ihrer formalen und inhaltlichen Unbestimmtheit (Implizitheit) gesehen werden. Der substantivierte Infinitiv ist für die Rollen des Substantivs im Satz nur beschränkt geeignet. Ein wesentlicher inhaltlicher Vorteil der Ableitung auf -ung gegenüber dem substantivierten Infinitiv ist deren Fähigkeit, eine umgrenzte Größe zu

18 Ähnliche Ergebnisse führt auch Wellmann 1975: 245 für sein Korpus an (50.2\% von 4157), das aber im wesentlichen aus anderen Textsorten und Quellen besteht als unser Korpus.

19 Dieses Korpus wurde aus 15 Diskussionen der Textbücher Heutiges Deutsch 1971 und 1974 sowie der Diskussion zum Vortrag von K.H. Bausch (1971) zusammengestellt (vgl. Petrič 1991 bzw. Petric 1993).

20 Ähnliche Anteile der Ableitung auf -ung in FVG zeigt auch das Korpus von So 1991, das auf populärwissenschaftlicher Gebrauchsprosa beruht: In der Epoche zwischen 1838 und 1980 betrug ihr durchschnittlicher Anteil in FVG etwa 36\% (vgl. So 1991: 259).

So 1991: 259 .

Vgl. dazu So 1991: 263.

v. Polenz 1963: 25, übernommen von So 1991: 261. 
bezeichnen, was ja als ein typisch nominales Merkmal angesehen werden kann. ${ }^{25}$ Unter den markiertesten Substantiven sind die Ableitungen auf -ung also aufgrund ihrer inhaltlichen und formalen Merkmale gegenüber den substantivierten Infinitiven als weniger markierte Substantive einzustufen. ${ }^{26}$ Die Fähigkeit der Ableitungen auf -ung, eine umgrenzte Größe zu bezeichnen, wird nun unter anderem in der deutschen Gegenwartssprache intensiv dazu genutzt, terminative FVG zu bilden. ${ }^{27}$ Da FVG als verstärkte Konstruktionen anzusehen sind, treten sie in Texten häufiger mit terminativer (d.h. inchoativer oder egressiver) Aktionsart auf und seltener mit kontinuativer Aktionsart. Terminative Aktionsarten sind markierter als kontinuative Aktionsarten, denn in den Sprachen der Welt ist die Existenz grammatikalisierter Konstruktionen zum Ausdruck von Kontinuitivität verbreiteter als die Existenz solcher Konstruktionen zum Ausdruck von Terminativität. Ein weiteres Argument für die größere Markiertheit der terminativen Aktionsarten wäre das (zusätzliche) semantische Merkmal der Begrenztheit, das die kontinuativen Aktionsarten nicht zum Ausdruck bringen. Eine andere Fähigkeit der Verbalabstrakta auf -ung, die aus transitiven Basisverben abgeleitet sind, ist ihre Unbestimmtheit in Bezug auf das Genus verbi. Diese Möglichkeit läßt in Abhängigkeit vom kombinierten FV (NV) die Bildung aktivischer oder passivischer FVG (NVG) zu. Gerade die passivischen FVG (z.B. Verwendung finden) haben sich in den letzten 150 Jahren als produktiver Bildungstyp erwiesen. $^{28}$

\section{NOMINALISIERUNGEN ALS KOMPLIZIERTE NOMINAL- PHRASEN}

Im Vergleich zu anderen Substantivgruppen, die kein Verbalabstraktum als Gliedkern enthalten, sind Nominalisierungen mit Verbalabstraktum als Gliedkern kompliziertere syntaktische Konstruktionen. ${ }^{29} \mathrm{Da}$ Verbalabstrakta $\mathrm{zu}$ den kompliziertesten Substantiven gehören, erwarten wir, daß auch die Phrasen, die sie bilden, komplizierte Konstruktionen sind, und zwar in Bezug auf ihre Struktur, Satzgliedschaft, Stellung im Satz und Wichtigkeit in der Mitteilungsperspektive (Thema-Rhema-Gliederung):

Schäublin 1972: 28-29.

Zur Markiertheit des Infinitivs in den europäischen Sprachen vgl. auch Mayerthaler/Fliedl/Winkler 1993.

Vgl. Leiss 1992: $255 \mathrm{ff}$.

So 1991: 246f. Der gemeinsame Nenner der FVG, ob nun aktivisch oder passivisch, ist ihre holistische Verbsemantik (Leiss 1992: 255).

In Petrič 1990 bzw. Petrič 1992 wurden aus der Annahme, Nominalisierungen seien komplizierte Nominalphrasen, Vorhersagen abgeleitet und mit statistischen Mitteln geprüft. Die Mehrzahl unserer Vorhersagen wurde bestätigt. 
- So weisen die Nominalisierungen häufiger markiertere Rechts-Attribute auf (z.B. Genitive, Präpositionalgruppen, Nebensätze, Infinitvsätze).

- Die Nominalisierungen bezeichnen etwas Unbelebtes und mit den Sinnesorganen nicht Faßbares. ${ }^{30}$ Belebtes ist unseren Sinnesorganen greifbar und steht dem prototypischen Sprecher näher. Unbelebtes kann von diesem Standpunkt aus als markierter gelten. ${ }^{31}$ Das unmarkierteste Satzglied (in Nominativ-Akkusativsprachen) ist das Subjekt. Die Nominalisierungen treten häufiger als Objekte (insbesondere als Akkusativobjekte) und Adverbialbestimmungen auf.

- Die Nominalisierungen (insbesondere jene mit einer Ableitung auf -ung als Gliedkern) kommen häufiger in Nebensätzen vor als in Hauptsätzen. Nebensätze sind ihren syntaktischen und illokutiven Rollen zufolge keine prototypischen Sätze und werden als markierter angesehen.

- Die Nominalisierungen treten im Vergleich zu anderen Nominalphrasen häufiger als Rhema des Satzes auf. Das Rhema kann als markierter eingestuft werden.

In unseren Korpora fällt auf, daß der Anteil von (markierteren) Rechts-Attributen in FF (etwa $25 \%$ von 275$)^{32}$ oder in NVG (etwa $36 \%$ von 443 ) größer ist als in FVG (etwa $16 \%$ von 132). ${ }^{33}$ Dieser Befund entspricht nicht unserer ursprünglichen Annahme, nach der die Zahl der postpositiven Attribute in FVG größer sein sollte als in NVG oder FF. So ${ }^{34}$ konnte in den Texten populärwissenschaftlicher Gebrauchsprosa nachweisen, daß der Anteil der vorangestellten Attribute in FVG in den letzten 150 Jahren abgenommen (von rund $97 \%$ im 17./18. Jahrhundert auf etwa $81 \%$ ), während der Anteil der markierteren nachgestellten Attribute in FVG deutlich zugenommen hat (von knapp 3\% im 17./18. Jahrhundert auf rund 17\%). Hinzukommt außerdem, daß der Anteil der mehrstufigen Attribution in den letzten 150 Jahren im Vergleich zum 17. und 18. Jahrhundert ebenfalls zugenommen hat, denn mehr als $90 \%$ solcher Fälle stammen aus der Zeit zwischen 1838-1980. ${ }^{35}$ Wiederum besteht ein Zusammenhang zu den Ableitungen auf -ung, die ja häufig von transitiven (also markierteren) Basisverben

$30 \quad$ Vgl. Mayerthaler 1981: 17.

31 Vgl. Mayerthaler 1981:14.

32 Dieses Korpus wurde lediglich aus dem Theaterstück 'Die Fische' von Peter Hacks gebildet. Vgl. auch Sommerfeldt 1969: 291, der in einem Vergleich zwischen 2000 Nomina actionis und 20000 Substantivgruppen ebenfalls zu dem Ergebnis kommt, daß die Nominalisierungen eine kompliziertere Attributstruktur aufweisen. Nach Sommerfeldt 1969: 291 zwingt die verbale Leistung der Gliedkerne dazu, das Subjekt oder Objekt der Verbhandlung als Attribut hinzusetzen. Auch Umstandsangaben können sich einstellen.

33 Die beiden letzten Angaben stammen aus dem Korpus in Petrič 1991 bzw. 1993, das aus 15 Diskussionen zusammengestellt worden ist. Eine Tendenz zur Attributlosigkeit in FVG stellt auch Wellmann 1975: 210 in seinem umfangreicheren Korpus fest.

34 So 1991: 226ff.

35 Vgl. So 1991: 230. 
gebildet werden. Daß unser Befund nicht dem in Sos Korpus entspricht, könnte mit dem größeren Anteil lexikalisierter Verbalabstrakta in unserem zweiten Korpus zusammenhängen.

\section{NOMINALISIERUNGEN ALS GESCHWÄCHTE KON- STRUKTIONEN}

Nominalisierungen mit einem nichtlexikalisierten Verbalabstraktum als Gliedkern sind im Vergleich zu den entsprechenden Nebensätzen geschwächte Konstruktionen, ${ }^{36}$ denn die syntaktischen und semantischen Beziehungen zwischen den Teilen einer Nominalisierung sind weniger deutlich als die entsprechenden Beziehungen zwischen den einzelnen Teilen des entsprechenden Satzes mit finter Verbform:

- Während man mit einer finiten Verbform die Kategorien Person, Numerus, Tempus, Modus und Genus verbi zum Ausdruck bringen kann, ist dies mit einer Nominalisierung nur beschränkt (mit anderen Mitteln) möglich.

- Die Nominalisierungen können die Verwendung nebensatzeinleitender Konjunktionen ersparen. Sie können aber in anderen Fällen auch von Präpositionen regiert werden, die den subordinierenden Konjunktionen der Nebensätze entsprechen.

- Die verbalen Ergänzungen sind z.T. syntaktisch obligatorisch, während die entsprechenden Ergänzungen eines Verbalabstraktums nur selten syntaktisch obligatorisch sind und daher in Texten häufig nicht ausgedrückt werden.

(26) Ich mache Sie für die Einhaltung verantwortlich. ${ }^{37}$

(27) Ich mache Sie dafür verantwortlich, daß die Soldaten den Befehl einhalten / daß der Befehl eingehalten wird.

- Die Nominalisierungen bezeichnen etwas Unbelebtes und treten daher häufig als Akkusativobjekt auf. Das unmarkierteste Satzglied zur Bezeichnung des Unbelebten ist (zumindest in den Nominativ-Akkusativ-Sprachen) das direkte Objekt.

- Die Nominalisierungen treten häufiger als Thema auf. Da das Thema an etwas anknüpft, was bereits zum Hintergrundwissen der Sprachteilnehmer gehört oder sogar explizit vorerwähnt ist, kann es gegenüber dem Rhema als unmarkiert eingestuft werden.

36 In Petrič 1990 bzw. Petrič 1992 wurden aus der Annahme, Nominalisierungen seien geschwächte Konstruktionen, Vorhersagen abgeleitet und mit statistischen Mitteln geprüft. Ein Teil unserer Vorhersagen wurde bestätigt.

37 Aus P. Hacks Theaterstück 'Die Fische' (S. 191). 
Als geschwächte syntaktische Konstruktionen sind die Nominalisierugen ein Mittel zur Vereinfachung der Äußerungsstruktur. In der deutschen Gegenwartssprache wirkt eine Tendenz zur Linearisierung der Satzstruktur. ${ }^{38}$ Diese Tendenz bewirkt eine Zunahme von Substantivgruppen und Parataxe und gleichzeitig eine Abnahme der Hypotaxe. Besonders stark ist der zahlenmäßige Rückgang von Nebensätzen zweiten und höheren Grades. ${ }^{39}$ Wir könnten daher annehmen, daß die Tendenz zur Linearisierung der Satzstruktur in Nebensätzen stärker wirkt als in Hauptsätzen, besonders stark jedoch in Nebensätzen, von denen ein oder mehrere weitere Nebensätze abhängig sind. ${ }^{40}$ Die Einebnung einer unübersichtlichen, komplexen Äußerungsstruktur durch Nominalisierung von Nebensätzen (zweiten und höheren Grades) erleichtert dem Sprecher das Produzieren von inhaltlich komplexen Äußerungen. Dem Hörer bzw. Leser liegt zwar eine einfachere (linearere) Äußerungsstruktur vor, aber dafür muß er mehr Denkarbeit investieren, um die meist fehlenden Ergänzungen und nur implizit oder im Kontext der Nominalisierungen ausgedrückten syntaktischen Beziehungen zu erschließen. Sind die Nominalisierungen nicht zu umfangreich und nicht zu dicht gestaffelt, leidet der Verstehensprozeß gewöhnlich nicht. Zumindest in Gesprächsabläufen kann das Fehlende meist relativ leicht aus dem Kontext bzw. aus dem Weltwissen erschlossen werden. Daher stellt eine verzweigte hypotaktische Struktur wohl ein größeres Dekodierungsproblem dar als eine geradlinigere Äußerungsstruktur mit einer Nominalisierung. Gestört wird dieses Gleichgewicht zwischen optimaler Sparsamkeit im Ausdruck und optimaler Deutlichkeit im Ausruck allerdings in einigen Funktionalstilen (z.B. in der Gesetzessprache, Publizistik, Wissenschaftssprache), in denen sich die Nominalisierungen häufen und mit zahlreichen und komplexen Attributen überfrachtet werden. Diese Funktionalstile sind jedoch nicht $\mathrm{zu}$ den natürlichen Gesprächsabläufen zu zählen. Die angenommenen Markiertheitsverhältnisse sind hier zum Teil wahrscheinlich nicht gültig. Eine Häufung komplexer Nominalisierungen dürfte jedenfalls für den Textproduzenten ein größeres Kodierungsund für den Textrezipienten ein wohl noch größeres Dekodierungsproblem darstellen als komplexe mehrstufige hypotaktische Satzstrukturen.

\section{FUNKTIONSVERBGEFÜGE ALS VERSTÄRKTE KON- STRUKTIONEN}

Treten Nominalisierungen jedoch in FVG (NVG) auf, verschmelzen sie mit dem FV zu einer neuen Bedeutungseinheit, in der sie den Hauptbedeutungsträger darstellen.

Vgl. Admoni 1973:79f. 
Die FVG (NVG) sind im Vergleich zu den entsprechenden Verben als verstärkte Konstruktionen zu werten. Sie sind meist formal aufwendiger, bedeutungsenger, schwieriger $\mathrm{zu}$ produzieren, jedoch leichter dekodierbar und behaupten sich vorzugsweise in komplizierten grammatischen Umgebungen. ${ }^{41}$

Die größere semantische und formale Komplexität der FVG zeigt sich in verschiedenen Merkmalen: z.B.

- Im Vergleich zu den Verben in unserem Kontrollsample sind die Basisverben der FN häufiger komplizierter gebaut, lang und seltener vorkommende Lexeme mit relativ wenigen Bedeutungsvarianten.

- Im Vergleich zu den Substantiven in unserem Kontrollsample sind die FN häufiger kompliziertere Wortbildungskonstruktionen, seltener vorkommende Lexeme mit relativ wenigen Bedeutungsvarianten.

- Im Vergleich zu den Nominalphrasen unseres Kontrollsamples sind die Nominalphrasen mit FN als Gliedkern häufiger Rhema des Satzes.

- Die Valenzmuster des FN und des FV kombinieren sich zu neuen verbalen Valenzmustern, die meist komplexer sind als die der entsprechenden einfachen Verben. Die Kreuzung der verbalen und nominalen Valenzmuster in FVG sehen wir als Folge der übergeneralisierenden Verwendung von häufigen und bedeutungsweiten Verben an.

(28) Er kommt in die Stadt. kommen (sub lok) ${ }^{42}$

(29) Der Stoff beruihrt Wasser. berühren (sub akk)

(30) Der Stoff kommt in Berührung mit Wasser.

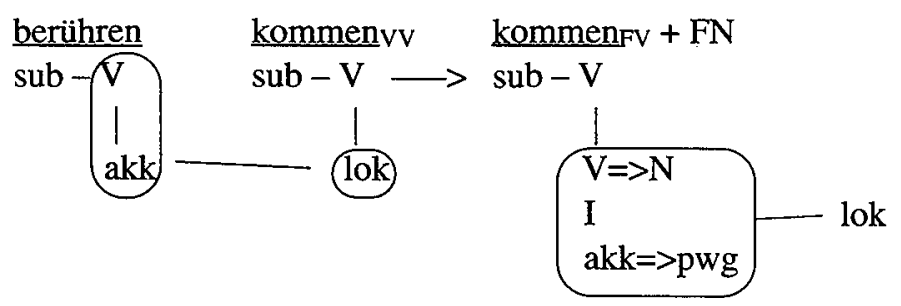

(31) Wir bringen Sie in die Stadt. bringen (sub akk lok)

(32) Wir verbinden Sie mit Herrn Krause. verbinden (sub akk prp)

(33) Wir bringen Sie in Verbindung mit Herrn Krause.

41 In Petrič 1991 bzw. Petrič 1993 wurden aus der Annahme, Nominalisierungen seien geschwächte Konstruktionen, Vorhersagen abgeleitet und mit statistischen Mitteln geprüft. Ein großer Teil unserer Vorhersagen wurde bestätigt.

42 In diesen Dependenzdarstellungen ist $s u b=$ Subjekt, $a k k=$ Akkusativergänzung, $l o k=$ (situative/ direktive) Lokalergänzung, $p r p=$ Präpositionalergänzung, $p w g=$ präpositionale Wortgruppe, $V V=$ Vollverb. 


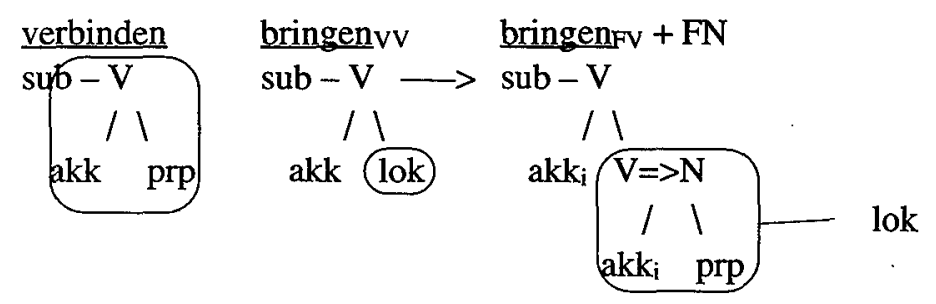

Durch die Reinterpretation von FV und FN zu einem Vollverb werden meist auch die Attribute des FN zu Satzgliedern umkategorisiert.

- Die FVG kommen häufiger in markierter Umgebung vor, d.h. in Nebensätzen, Infinitivsätzen, in Sätzen mit einem FV in der nichtdritten Person, im Plural und im Nicht-Präsens.

In FVG (meist auch in NVG) gehen meistens bereits lexikalisierte oder sich lexikalisierende Verbalabstrakta, unter denen viele zum allgemeinwissenschaftlichen Wortschatz ${ }^{43}$ gehören, Verbindungen mit denotativ bedeutungsschwachen Verben ein. Die Lexikalisierung der Fügungen ist verschieden stark ausgeprägt und übt auch auf die nominalen Eigenschaften der darin vorkommenden Verbalabstrakta ihren Einfluß aus. Im Fehlen bestimmter nominaler Eigenschaften des Verbalabstraktums sehen wir eine mehr oder weniger stark ausgeprägte Tendenz, sich an die verbale Umgebung anzupassen, d.h erneut verbale Eigenschaften aufzunehmen: ${ }^{44}$

a) Das FN eines FVG ist nicht anaphorisierbar.

b) Der Artikelgebrauch in einem FVG ist eingeschränkt.

c) Die Pluralfähigkeit eines FN geht verloren.

d) Die Zahl als auch die Art der Attribute ist eingeschränkt.

e) Attribute können zu Satzgliedern umfunktioniert werden.

f) Ein FVG kann durch ein entsprechendes Vollverb (Adjektiv) substituiert werden, denn es bildet eine Bedeutungseinheit.

g) Zwischen einem FVG und dem entsprechenden Vollverb besteht aber gewöhnlich ein Unterschied in der Aktionsart (Aspekt).

h) Die Negation erfolgt nicht wie bei Substantiven üblich bzw. möglich mit dem Negationswort kein, sondern mit der Negationspartikel nicht.

i) Die Satznegation nicht und adverbiale Bestimmungen können nicht zwischen FN und FV treten.

j) Das FN wird in die verbalen Stellungsfelder des Satzes eingegliedert.

k) Die Valenzmuster des FN und des FV kombinieren sich zu neuen, komplexeren verbalen Valenzmustern.

Vgl. Köhler 1985: 27.

44 Die folgende Merkmalliste der FVG stützt sich auf Helbig 1979:276f. Wir führen keine Beispiele dazu an, da diese leicht der einschlägigen Fachliteratur entnommen werden können. 
1) FN und FV kommen gewöhnlich in Kommutationsreihen vor.

Die vorgeführten Merkmale a) - 1) sind je nach Festigkeit (Lexikalisierung) der Fügung mehr oder weniger ausgeprägt. Im Gegensatz zu den FVG weisen die NVG im allgemeinen eine geringere Festigkeit auf. Daher treffen viele der angeführten Merkmale nicht auf sie zu.

Während die Verbalabstrakta in freien Fügungen sich in Richtung Nomen entwickeln, d.h. im Prozeß der Lexikalisierung zunehmend nominale Eigenschaften erhalten, entwickeln sich die Verbalabstrakta in FVG nicht mehr in Richtung Nomen, sondern eher in den verbalen Bereich hinein. Dies wird auch daran sichtbar, daß FN mit ihren Präpositionen und FV, nachdem die Lexikalisierung des Gefüges bereits weit fortgeschritten ist, zu einem Wort, nämlich zu einem Verb, verschmelzen können, in dem das FN zusammen mit der Präposition die erste unmittelbare Konstituente des neuen Verbs darstellt:

(34) zu Stande kommen $\rightarrow$ zustandekommen.

Daß die Teile eines FVG sowohl syntaktisch als auch semantisch eine Einheit billden und daß das von einem Sprachteilnehmer auch so aufgefaßt wird, wird durch die Zusammenschreibung natürlich auf auffällige Weise veranschaulicht: sowohl die Bedeutung des FN (ein Geschehen) als auch die Bedeutungen der Präposition (eine abstrakte Richtung, die als Zeitrichtung aufgefaßt wird) und des FV (die Aktionsart) sind nun in einem Wort vereint. ${ }^{45}$

Unserer Grundannahme folgend, daß FVG verstärkte Konstruktionen sind, würden wir erwarten, daß auch das zentrale Wort einer verstärkten Konstruktionen (im FVG ist dies das FN) eine komplizierte Form ist. Diese Annahme hat sich bestätigt. In der weiteren Entwicklung der FVG können nach unserer Annahme aber auch weniger markierte Verbalabstrakta als zentrales Wort einer FVG auftreten. Das Korpus von So scheint so eine Entwicklung zu zeigen. In den von ihm analysierten Texten aus dem 17. und 18. Jahrhundert kommen die (als Nomina) markierten substantivierten Infinitive häufiger als FN vor, in den Texten des 19. und 20. Jahrhundert hingegen die wegen ihrer typisch nominalen Fähigkeit, eine begrenzte Größe bezeichnen zu können, weniger markierten Ableitungen auf -ung. Mit voranschreitender Grammatikalisierung der umgedeuteten Gefüge wurden die Ableitungen auf -ung und die FV als semantische Einheit angesehen. Das bei vielen Ableitungen auf -ung auftretende Merkmal der Begrenzheit wurde in verbaler Umgebung vielleicht als terminativ und damit als markiert empfunden. ${ }^{46}$

45 Nach dem Lüdtkeschen Prinzip der Verschmelzung werden sprachliche Einheiten, die sehr häufig nebeneinander vorkommen, vom Sprachteilnehmer nicht mehr als segmentiert wahrgenommen, sondern schließlich als eine einzige sprachliche Einheit erlebt (Lüdtke 1979: 15). Die Kodierung von Kognitiv Zusammengehörendem in einer Form sehen wir als unmarkierte Option an. 
Die äußere Motivierung für die größere Verbreitung von FVG und der Entstehung neuer FVG scheint die rasante gesellschaftliche Entwicklung in den letzten 150 Jahren gewesen zu sein, insbesondere aber im Bereich der Wissenschaft, Technik, Publizistik und Verwaltung, in denen die schon lange bereitstehenden Konstruktionen wegen des charakteristischen Strebens nach Kürze und Prägnanz einen natürlichen Nährboden fanden. Der Sprachgebrauch in diesen Lebensbereichen beeinflußt seitdem auch den Sprachgebrauch in anderen Lebensbereichen maßgeblich.

Da die FVG nicht erst seit 150 Jahren bestehen, sondern bereits im Ahd. nachweisbar sind ${ }^{47}$ ist es wichtig, eine sprachinterne Motivierung für die Entstehung von FVG zu ergründen. Die sprachinterne Motivierung für die Entstehung von FVG im Deutschen ist wohl die von fast allen Linguisten an erster Stelle genannte Möglichkeit, die Verbhandlung bezüglich der Aktionsarten genauer zu bestimmen oder wie Leiss ${ }^{48}$ annimmt, im deutschen Verbalsystem erneut Aspektpaare zu bilden. In Sprachen, die über ein voll ausgebildetes Aspektsystem verfügen (z.B. die slawischen Sprachen) und dennoch Fügungen aufweisen, die den deutschen FVG ähneln, muß allerdings zumindest primär eine andere Motivierung vorliegen. Vermutlich hängt die Entstehung und Verbreitung der FVG in diesen Sprachen eher mit anderen (nominalen) Leistungen der FVG und der Nominalisierungen überhaupt zusammen.

\section{LITERATUR}

ADMONI, WLADIMIR (1973): Die Entwicklungstendenzen des deutschen Satzbaus von heute. Linguistische Reihe Band 12. Max Hueber Verlag. München 1973.

BENEŠ, EDUARD (1981): Die formale Struktur der wissenschaftlichen Fachsprachen in syntaktischer Hinsicht. In: BUNGARTEN, THEO (Hg.) (1981): Wissenschaftssprache. Wilhelm Fink Verlag. München 1981. S. 185-212.

ten CATE, ABRAHAM P. (1985): Aspektualität und Nominalisierung. Zur Bedeutung satzsemantischer Beziehungen für die Beschreibung der Nominalisierung im Deutschen und im Niederländischen. Peter Lang. Frankfurt a.M. 1985.

DANIELS, KARLHEINZ (1963): Substantivierungstendenzen in der deutschen Gegenwartssprache. Schwann. Düsseldorf 1963.

DISKUSSION zum Vortrag von Karl-Heinz Bausch (1971). In: Forschungen zur gesprochenen Sprache und Möglichkeiten ihrer Didaktisierung. Protokoll eines Werkstattgesprächs des Goethe-Instituts am 10. und 11. Dezember 1970; veranstaltet vom Referat für Technische Unterrichtsmittel in Zusammenarbeit mit der Forschungsstelle Freiburg des Instituts für Deutsche Sprache Mannheim.

$47 \quad$ Vgl. Relleke 1974.

48 Leiss 1992: 255ff. 
Herausgegeben vom Goethe-Institut, Referat für Unterrichtstechnologie und Mediendidaktik. München.

ENGELEN, BERNHARD (1968): Zum System der Funktionsverbgefüge. In: Wirkendes Wort 18 (1968), S. 289-303.

HELBIG, GERHARD (1979): Probleme der Beschreibung von Funktionsverbgefügen im Deutschen. In: DaF 5/1979, S. 273-285.

HERINGER, HANS-JÜRGEN (1968): Die Opposition von 'kommen' und 'bringen' als Funktionsverben. Sprache der Gegenwart Band III. Pädagogischer Verlag Schwann. Düsseldorf 1968.

HEUTIGES DEUTSCH II/1 (1971): Texte gesprochener deutscher Standardsprache I. IDS Mannheim Forschungsstelle Freiburg i. Br. Max Hueber Verlag München / Pädagogischer Verlag Schwann Düsseldorf.

HEUTIGES DEUTSCH II/2 (1974): Texte gesprochener deutscher Standardsprache II. IDS Mannheim Forschungsstelle Freiburg i. Br. Max Hueber Verlag München / Pädagogischer Verlag Schwann Düsseldorf.

JIE, YUAN (1986): Funktionsverbgefüge im heutigen Deutsch. Eine Analyse und Kontrastierung mit ihren chinesischen Entsprechungen. Julius Groos Verlag. Heidelberg 1986.

KÖHLER, CLAUS (1985): Verben in deutschsprachigen Fachtexten -Supplementverben (eine Voraussetzung der Nominalität von Fachtextsätzen). Schriftenreihe Fachsprache - Fremdsprache -Muttersprache, TU Dresden, Heft 1.

LEISS, ELISABETH (1992): Die Verbalkategorien des Deutschen. Ein Beitrag zur Theorie der sprachlichen Kategorisierung. Walter de Gruyter. Berlin, New York 1992.

LÜDTKE, HELMUT (1979): Sprachwandel als universales Phänomen. In: Lüdtke, Helmut (1979) (Hg.): Kommunikationstheoretische Grundlagen des Sprachwandels. Walter de Gruyter. Berlin, New York 1979.

MAYERTHALER, WILLI (1981): Morphologische Natürlichkeit. Athenaion. Wiesbaden 1981.

MAYERTHALER, WILLI / FLIEDL, GÜNTHER / WINKLER, CHRISTIAN (1993): Infinitivprominenz in europäischen Sprachen. Teil I: Die Romania (samt Baskisch). Gunter Narr Verlag. Tübingen 1993.

OREŠNIK, JANEZ (1990a): Periphrasen sind verstärkte Konstruktionen. In: Boretzky N./Enninger W./ Stolz T. (1990) (Hgg.): Spielarten der Natürlichkeit - Spielarten der Ökonomie. Beiträge zum 5. Essener Kolloquim über "Grammatikalisierung: Natürlichkeit und Systemökonomie" vom 6.10.-8.10.1988 an der Universität Essen. Zweiter Band, erster Halbband, S. 85-99.

OREŠNIK, JANEZ et alii (1990b): Introduction to the Subsequent Three Papers in the Present Volume. In: LINGUISTICA XXX, S. 512. Ljubljana 1990.

PETRIČ, TEODOR (1990): Posamostaljenja v knjižni nemščini. <Nominalisierungen in der deutschen Standardsprache.> Magisterarbeit. Ljubljana 1990. 
PETRIČ, TEODOR (1992): Nominalisierungen sind geschwächte syntaktische Konstruktionen. (erscheint voraussichtlich in: LINGUISTICA 35/1995, Ljubljana). Manuskript.

PETRIČ, TEODOR (1991): Funktionsverbgefüge als verstärkte syntaktische Konstruktionen. Manuskript eines Referats anläßlich der 19. Arbeitstagung österreichischer Linguisten (Klagenfurt, 25.-27.10.1991).

PETRIČ, TEODOR (1993): Funktionsverbgefüge und Nominalisierungsverbgefüge im Deutschen als verstärkte syntaktische Konstruktionen im Vergleich zu stammgleichen Verben und Adjektiven. (erscheint voraussichtlich in: Papiere zur Linguistik 2/1993). Manuskript.

von POLENZ, PETER (1985): Deutsche Satzsemantik. Grundbegriffe des Zwischenden-Zeilen-Lesens. Walter de Gruyter. Berlin, New York 1985.

von POLENZ, PETER (1987): Funktionsverben, Funktionsverbgefüge und Verwandtes. Vorschläge zur satzsemantischen Lexikographie. In: ZGL 15 (1987), S. 169-189.

RELLEKE, WALBURGA (1974): Funktionsverbgefüge in der althochdeutschen Literatur. In: Amsterdamer Beiträge zur älteren Germanistik 7 (1974), S. 1-46.

SO, MAN-SEOB (1991): Die deutschen Funktionsverbgefüge in ihrer Entwicklung vom 17. Jahrhundert bis zur Gegenwart. Eine sprachhistorische Untersuchung anhand von populärwissenschaftlichen Texten. WVT Wissenschaftlicher Verlag. Trier 1991.

SCHÄUBLIN, PETER (1972): Probleme des adnominalen Attributs. Morphosyntaktische und semantische Untersuchungen. Studia Linguistica Germanica 5. Walter de Gruyter. Berlin New York 1972.

SOMMERFELDT, KARLERNST (1969): Zur Struktur der Substantivgruppe in einigen funktionalen Stilen. In: Deutsch als Fremdsprache 1969/5, S.287-295.

TOMAN, JINDRICH (1987): Wortsyntax. Eine Diskussion ausgewählter Probleme deutscher Wortbildung. Max Niemeyer Verlag. Tübingen 1987.

WELLMANN, HANS (1975): Deutsche Wortbildung - Typen und Tendenzen in der Gegenwartssprache, 2. Hauptteil: Das Substantiv. Pädagogischer Verlag Schwann. Düsseldorf 1975.

Povzetek

O NEKATERIH STRUKTURALNIH LASTNOSTIH POSAMOSTALJENJ V NEMŠČINI

Kot v mnogih knjižnih jezikih je v nemščini opazna težnja $k$ posamostaljevanju stavčnih vsebin. V članku se omejujemo na posamostaljenja, ki imajo za jedro izglagolsko izpeljanko $\mathrm{z}$ abstraktnim pomenom. $\mathrm{V}$ nemščini je moč razlikovati vsaj dve pojavni obliki: 1 . nevezana posamostaljenja $v$ tki. prostih zgradbah (freie Fügungen), tj. $v$ tki. frazeoloških glagolskih zvezah (Funktionsverbgefüge). V članku želimo predstaviti tele domneve: Posamostaljenja se lahko razvijejo $v$ dve različni smeri. Nevezana posamostaljenja sčasoma izgubijo glagolske lastnosti (npr. prvotno vezljivost) in z napredujočo leksikalizacijo pridobijo čedalje več samostalniških lastnosti (npr. množino). V primerjavi z ustreznimi odvisnimi stavki so posamostaljenja ošibljene zgradbe, kajti skladenjska 
in pomenska razmerja med posameznimi deli posamostaljenja niso tako jasna kot med posameznimi deli odvisnih stavkov. V manj zaznamovanih besedilih so nevezana posamostaljenja zgradbeno manj zapletena kot ustrezni odvisni stavki, za tvorca besedila glede na zaželeno natančnost lažje ubesedljiva in za naslovnika besedila zaradi manjše obvestilnosti težje razumljiva. V frazeoloških glagolskih zvezah izgubijo izglagolske izpeljanke samostalniške lastnosti in pridobijo $\mathrm{z}$ napredujočo leksikalizacijo zgradbe več glagolskih lastnosti. V primerjavi $\mathrm{z}$ ustreznimi glagoli so te opisne zgradbe okrepljene skladenjske zgradbe, kajti zgradbeno in pomensko so bolj zapletene, za tvorca besedila so glede na zaželeno natančnost težje ubesedljiva in za naslovnika besedila zaradi večje obvestilnosti lažje razumljiva. 